\title{
WolStat: A new statistics package for the behavioral and social sciences
}

\author{
Allen H. Wolach \\ Illinois Institute of Technology, Chicago, Illinois \\ AND \\ Maureen A. Mchale \\ Northwestern State University, Natchitoches, Louisiana
}

\begin{abstract}
WolStat differs in several important ways from other statistics packages. Data are entered using a text editor, as opposed to a data grid. WolStat performs all possible simple to simple simple simple simple main effects tests and multiple comparison tests for ANOVAs (independent groups, split plot, and randomized block factorial) with up to five factors. More nonparametric statistics can be performed than with most other statistics packages. Latin squares can be randomly generated. Uniformly distributed random integers (with or without replacement) or normally distributed random numbers can be generated. WolStat provides a tutorial and assistance for selecting orthogonal comparisons. WolStat also provides general and specific models for fixed, mixed, and random ANOVAs with one through five factors.
\end{abstract}

WolStat is a statistics package that is available without cost. No license is required to use WolStat for statistics courses or for other courses requiring statistical analyses.

Data are entered in WolStat using a text editor (data editor) as opposed to a data grid. A data editor makes it possible to enter large data sets very rapidly.

An investigator can explicitly select any of 20 ANOVAs from a menu system. Investigators can perform ANOVAs with up to five factors. None, some, or all factors can be repeated (correlated) measures factors. The remaining factors are independent groups factors. Any factor can be designated as a fixed or random factor.

When WolStat is used to perform ANOVAs with up to five factors, it automatically provides simple main effects tests for statistically significant double interactions, simple simple main effects tests for statistically significant triple interactions, and so on. WolStat uses Tukey's procedure to perform all possible pairwise multiple comparison tests for each statistically significant main effect, simple main effects test, simple simple main effects test, and so on. Simple main effects tests, simple simple main effects tests, and Tukey's procedure are described in Kirk (1995).

WolStat performs over 30 nonparametric statistics, including a nonparametric analysis of covariance. It can generate uniformly distributed random integers with and without replacement. Normally distributed random numbers can also be generated. WolStat can randomly generate Latin squares with up to nine levels for each factor and can also be used to determine probability levels for a number of statistics. That is, it can be used as a replacement for statistics tables found at the back of statistics textbooks.
The output file for an ANOVA (and for all other statistics producing output files) is an ASCII (text) file that includes the ANOVA summary table for the selected alpha level, all possible main effects tests, simple main effects tests, and multiple comparison tests for the selected alpha level.

\section{Differences Between WolStat and Other Statistics Packages}

The data editor in WolStat makes it possible to enter large data sets in far less time than it takes to enter data in data grids found in other statistics packages. Data gridseven on fast computers-usually take some time to register an entry and place the cursor for the next entry.

Most statistics packages require a data file for all statistics being performed. Wolstat includes a number of statistics that never require more than six data entries. Statistics that require entry of six or fewer data points have data entered in text boxes on the monitor screen rather than to a data file.

Other statistics packages do not automatically perform simple main effects tests, simple simple main effects tests, or other such tests. For example, when an independent groups two-way ANOVA is performed by SPSS, an investigator can opt to display multiple comparison tests for the two main effects. However, the investigator cannot opt to display simple main effects tests (Kirk, 1995) or pairwise multiple comparison tests for the simple main effects tests for the rows and columns of the two-way interaction.

WolStat performs a larger number of nonparametric statistics than do SPSS and SAS. Unlike most statistics packages, WolStat automatically performs corrections for ties 
and for continuity for a given nonparametric statistic. Also, other statistics packages do not generate random numbers, Latin squares, or provide an alternative to a statistics table.

Since output files for WolStat are text files, an investigator can easily add comments and labels to a completed output file. An investigator does not have to learn a complicated and restricted system for changing default labels to labels with new names.

"Help" system. The comprehensive "Help" system for WolStat can be accessed from the menu at the top of any given WolStat screen. When a user is in the data editor, help is limited to that for the data editor. When the user is not in the data editor, help for all aspects of WolStat-except the data editor-is available from the "Help" menu.

The "Help" system provides information about data entry for each of the statistics in WolStat. Help for a given statistic also includes information about missing scores, as well as additional statistics performed on the data. WolStat comes with a file containing a comprehensive manual (MANUAL.RTF) on the WolStat CD. When WolStat is installed, the manual is placed in the program's directory on the hard drive. The manual can be printed using almost any word processor. The manual can also be installed on the hard drive. When the icon for the manual is clicked, the manual can be read on the monitor screen.

\section{THE DATA EDITOR}

\section{Example of Data Entry}

Entering data for a $t$ test. Suppose an investigator wants to perform an independent groups $t$ test. In order to minimize space taken by the following example, Group 1 is limited to four scores $(3,7,2,1)$, and Group 2 is limited to five scores $(5,8,9,8,7)$. The following data are entered in the data editor.

$$
\begin{aligned}
& 3,7,2,1,2 \mathrm{E} 22 \\
& 5,8,9,8,7,2 \mathrm{E} 22
\end{aligned}
$$

Figure 1 shows the top of the data editor screen with entries for the $t$ test. Note that the number 2E22 is used as a delimiter to indicate the end of observations for a group. The number 2E22 was chosen as a delimiter because it is larger than any number likely to be entered for a data point. Using a number instead of a string variable for a delimiter makes it possible to limit data files to numbers. Visual Basic can process files limited to numbers more rapidly than files with numbers and strings. Also note that numbers within a line of the data file are separated by a comma and a space. The last number in a line of the data file is not followed by a comma. The numbers for the $t$ test could have been entered in many other ways - for example,

$$
\begin{aligned}
& 3,7,2 \\
& 1,2 \mathrm{E} 22,5 \\
& 8,9,8,7 \\
& 2 \mathrm{E} 22
\end{aligned}
$$

\section{Simplifying Data Entry}

WolStat has a "Clean Data File on Exit" feature that allows a user to enter data without entering any or all of the commas in a data file. It also places spaces after commas, should the user fail to do so. For example, a user could enter

$$
\begin{aligned}
& 3,72 \\
& 12 \mathrm{E} 225 \\
& 89,87 \\
& 2 \mathrm{E} 22
\end{aligned}
$$

When the "Clean Data File on Exit" feature is selected, WolStat changes the preceding entries to the following:

$$
\begin{aligned}
& 3,7,2 \\
& 1,2 \mathrm{E} 22,5 \\
& 8,9,8,7 \\
& 2 \mathrm{E} 22
\end{aligned}
$$

Each time "Clean Data File on Exit" is selected, the data editor flags inappropriate data entries, one at a time. Suppose the letter $A$ is in a data file. Since the letter $E$ (upper or lowercase) is the only letter of the alphabet permitted in a data file, a message indicating that the letter $A$ should be removed appears on the screen.

\section{Simplifying Proofreading}

An "Easy Read Format for Data File" option places data in neat columns on the screen. For example, if "Easy Read Format for Data File" is selected after data for the $t$ test program are entered, the following appears on the screen:

\section{$37212 \mathrm{E} 22$}

\section{$589872 \mathrm{E} 22$}

If "Clean Data File on Exit" is selected, the data file is saved as follows:

$$
\begin{aligned}
& 3,7,2,1,2 \mathrm{E} 22 \\
& 5,8,9,8,7,2 \mathrm{E} 22
\end{aligned}
$$

\section{Menu System of the Data Editor}

Compatibility with word processors. The data editor is a full-featured editor with a text entry procedure similar to the text entry procedures for WordPad, Microsoft Word,

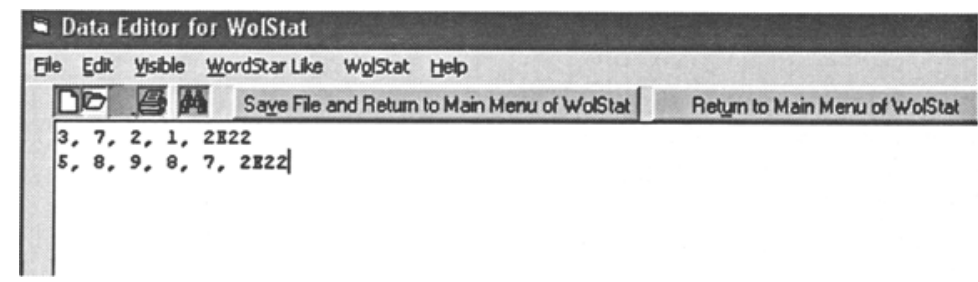

Figure 1. Data entry screen with $t$ test data. 
or WordPerfect. The data editor also supports most of the commands in the WordStar word processor. (Users who are not familiar with WordStar may ignore the WordStarlike commands.) The data editor produces ASCII (text) data files. When a user saves a data file, a standard Windows dialog box for entering a file name appears.

The "File" menu of the data editor. The "File" menu has standard options for a word processor: New, Open, Save, and Save As. WolStat also has options for inserting an entire file in a data file or saving a selected block of text to another file.

The "Edit" menu of the data editor. The "Edit" menu has standard options for Cut, Copy, Paste, Select All, Delete, Find, Find and Replace One at a Time, and Replace All.

The "Visible" menu of the data editor. The "Visible" menu has standard options to modify the way text appears on the screen. For example, the "Visible" menu has options for selecting fonts. In order to make it easy to read a data file on the screen, a font such as Courier New that takes the same space (width) for all numbers and characters is desirable.

\section{Selecting a Subset of Cases for Analysis}

Protecting the original data file. Since WolStat uses a data editor as opposed to a grid for data entry, it is easy to make a data file with a subset of cases. The original data file is saved (if it is not already saved).

Making a data file with a subset of cases. The original data file is brought into the data editor. The case (subset of data for a condition, treatment, group, etc.) that should be the first case is selected with the mouse. "Cut" and "Paste" are used to place the selected case at the top of the data file. The preceding process can be used to put the cases in any order.

Any cases not needed in the rearranged file can be selected with the mouse and deleted. Then "Save As" is used to save the file with a subset of cases. Now the user has two files: the original and the rearranged file. The preceding process can be repeated to make a series of data files containing subsets of data different from the original data file.

\section{MAIN MENU OF WOLSTAT}

\section{Running a Statistic}

Keyboard versus data file. There are two ways of entering data for statistics. Statistics never requiring entries for more than six observations have data entered in text boxes on the monitor screen. Statistics sometimes requiring more than six data entries use a data file.

Data file. Data for the $t$ test example were entered in a data file because the two groups could have many observations. After the investigator entered the data in the data editor and returned to the main menu of WolStat, the file was automatically opened. Of course, a user can choose another data file from the "Open" option of the "File" submenu of the main menu of WolStat.

Suppose the independent groups $t$ test is selected from the "Statistics" submenu of the main menu and the following data are in a data file:

\section{$3,7,2,1,2 \mathrm{E} 22$}

$$
5,8,9,8,7,2 \mathrm{E} 22
$$

WolStat provides a screen with options for eight data transformations and a default option of no data transformation. If the default option of no data transformation is selected, WolStat displays the output in Figure 2 on the monitor for the $t$ test example.

When output is on the monitor, an output file can be saved from the "File" menu of the main menu of WolStat. The output file is saved with the same name as the data file coupled with an extension of ".prt." Output files are text files and can be edited using almost any editor or word processor. If an investigator needs help related to what should be entered in a data file (or how to interpret output), the investigator can click on the "Help" menu of the main menu of WolStat. The "Help" menu provides an "Overview of WolStat," "General Instructions for Entering Data," and specific instructions for entering data for each of the statistics in WolStat.

\section{Statistics That Do Not Require a Data File}

Tabled values. WolStat can be used as a substitute for the statistics tables commonly found at the back of statistics text books. Suppose "Statistics|Tabled Values|t Values" is selected. WolStat provides a screen requesting entries for "Degrees of Freedom" and " $t$ Value" in text boxes. If the investigator enters 7 for "Degrees of Freedom" and 2.43 for " $t$ Value," the following output appears on the screen:

Probability for $t$ Value

Degrees of freedom $=7$

$t$ value from experiment $=2.43000$

Two-tailed probability $=0.04542$

One-tailed probability $=0.02271$

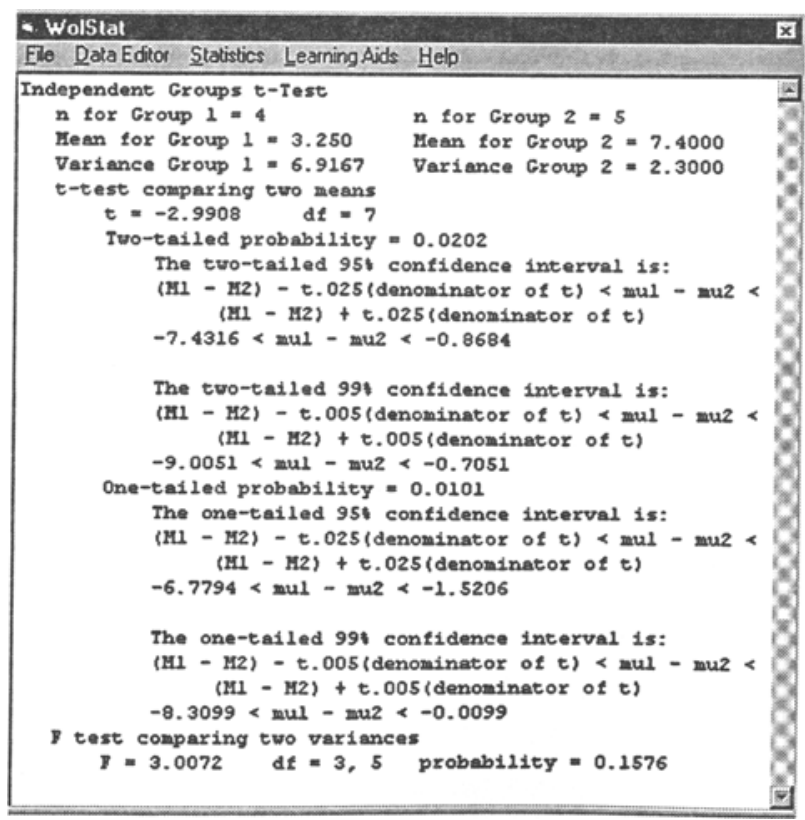

Figure 2. Output for independent groups $t$ test. 
Data files are not required for obtaining the probability for "Degrees of freedom $=7$ " and " $t$ value from experiment $=2.43000$."

WolStat provides other "Tabled Statistics Value" options that do not require a data file, such as $z$ values and height of distribution, $t$ values, chi-square values, $F$ values, and Mann-Whitney $U$ values.

Randomly generate Latin squares. WolStat can be used to randomly generate Latin squares. The investigator can choose to generate Latin squares ranging from $3 \times 3$ to $9 \times 9$. The Latin square appears on the monitor.

Random integers and random numbers. WolStat can generate random numbers. When "Random Numbers" is selected, the investigator can choose random integers with or without replacement or uniformly distributed normal numbers.

When "Random Integers With Replacement" is selected, the investigator enters the lowest integer (integer greater than or equal to 0 ), highest integer (integer greater than lowest number and less than 30,000), and the number of integers. WolStat responds with the appropriate number of integers in the appropriate range. When "Random Integers Without Replacement" is selected, the investigator enters the lowest integer (integer greater than or equal to 0 ), and highest integer (integer greater than lowest number and less than 30,000). WolStat responds with all integers in the appropriate range. Integers are not repeated.

Random uniform numbers are presented as integers because of the way most investigators in the behavioral and social sciences use a random number table. Suppose an investigator has 40 rats to assign to each of two groups. The investigator assigns consecutive integers (not randomly) to the rats by punching a binary code in a given rat's ear(s) with an ear punch. Then the investigator enters WolStat and selects random integers without replacement. The investigator enters 1 for lowest integer, 40 for highest integer, and 40 for number of integers. Wolstat provides the numbers 1 through 40 in a randomly determined order. Rats with numbers corresponding to the first 20 random integers are assigned to Group 1 , and rats with numbers corresponding to the remaining random integers are assigned to Group 2.

When "Normally Distributed Random Numbers" is selected, the investigator enters the mean of the distribution, standard deviation for the distribution, and number of randomly distributed random numbers. WolStat responds with normally distributed random decimal numbers in the appropriate range. Suppose the investigator enters 100 for mean of the distribution, 13 for standard deviation for distribution, and 25 for number of randomly distributed random numbers. WolStat responds with 25 numbers that are distributed much like scores on an intelligence test.

Nonparametric $2 \times 2$ and one-sample binomial test. Three of the statistics on the "Nonparametric $2 \times 2$ and One Sample Binomial Test" menu have data entered from the keyboard as opposed to a data file. These statistics are "Binomial Test," "Chi-square in a Two by Two Table," and "Poisson Distribution." Similarly, three of the statistics on the "Correlation" menu have data entered from the keyboard. These statistics are "Index of Agreement," "Tetrachoric Correlation Coefficient," and "Phi Coefficient."

\section{Statistics That Require a Data File}

General and specific instructions for entering data. The appropriate way to enter data for all statistics requiring a data file is provided by the list box found in the "Help" menu of the main menu of WolStat. The list item titled "General Instructions for Entering Data" explains how data are entered in WolStat. The list item for a specific statistic explains the order of data entry for the statistic.

\section{Description of Statistics Requiring a Data File}

Descriptive. The "Descriptive" menu item provides descriptive statistics for data from a series of groups. These statistics include $N$, mean, population standard deviation, sample standard deviation, standard error of the mean, average deviation, coefficient of variation, maximum, minimum, median, $Q$ (observation at the third quartile minus observation at the first quartile), range, skewness, kurtosis, mode, frequency distribution (when appropriate), and cumulative frequency distribution (when appropriate).

If a data transformation is required for the data for "Descriptive" statistics, WolStat provides an option for any of eight data transformations. The selected data transformation (or no data transformation) is performed on data for all groups. All statistics described above for "Descriptive" statistics are calculated using the transformed data.

The Appendix shows the statistics in WolStat that require data files.

Data transformations. The last column in the table in the Appendix shows whether a statistic permits or does not permit a data transformation. An investigator can choose one of eight data transformations (or no data transformation) for a statistic permitting a data transformation.

ANOVAs. The notation used for ANOVAs in WolStat requires some explanation. An independent groups ANOVA is sometimes referred to as a completely randomized (CR) ANOVA. An independent groups factorial ANOVA is sometimes referred to as a completely randomized factorial ANOVA. Consequently, an ANOVA designated "CR-p" is an independent groups ANOVA with one factor (p). An ANOVA designated "CRF-pqr" is an independent groups factorial ANOVA with three factors ( $p, q$, and $r)$.

Split plot factorial (SPF) ANOVAs have some independent groups factors and some repeated measures factors. A decimal point separates independent groups factors from repeated measures factors. A four-factor split plot factorial ANOVA with one independent groups factor (p) and three repeated (or correlated) measures factors ( $q, r$, and s) could be designated "SPF-p.qrs."

A randomized block (RB) ANOVA has one repeated (or correlated) measures factor (p). Since the factor is a repeated or correlated measures factor, it is placed after a decimal point (RB-.p). A randomized block factorial (RBF) ANOVA has repeated (or correlated) measures for all factors. All factors are placed after a decimal point to indicate that the factors are repeated or correlated measures factors. A randomized block ANOVA with three factors of interest could be designated "RBF-.pqr." The abbreviated notation for an ANOVA (e.g., CR-p) is very similar to the notation described by Kirk (1995) and by Winer, Brown, and Michels (1991). 
An investigator can choose to have $F$ ratios calculated for a fixed, mixed (assuming the ANOVA has at least two factors), or random model for a given ANOVA. The investigator also supplies the alpha level for the ANOVA. If quasi- $F$ ratios are required for the model, the investigator can choose between Cochran's (1951) method or Satterthwaite's (1946) method for calculating quasi- $F$ ratios. WolStat automatically performs simple main effects tests, simple simple main effects tests, and so on for any statistically significant interaction. Tukey's procedure is used to make pairwise multiple comparisons for any statistically significant main effect, simple main effect, simple simple main effect, and others. Data for the simple main effects tests, simple simple main effects tests (among others), and Tukey's procedure are transformed with the same data transformation (or no transformation) used for the ANOVA. If the Latin square ANOVA (LS-p) is chosen, a one-factor Latin square ANOVA can be performed. WolStat does not automatically perform simple main effects tests, simple simple main effects tests, and so on for statistically significant LS-p ANOVA interactions. Multiple comparison tests are not automatically performed.

ANOCOVAs. The abbreviated notation for an ANOCOVA (e.g., CRAC-p) is very similar to the notation in Kirk (1995). ANOCOVAs are performed using a fixed effects model. Data transformations can be performed on the variable of interest, but not on the covariate. WolStat does not automatically perform simple main effects tests, simple simple main effects tests, and so on for statistically significant ANOCOVA and Latin square ANOCOVA interactions. Multiple comparison tests are not automatically performed. If a Latin square ANOCOVA (LSAC-p) is chosen, a one-factor Latin square ANOCOVA with one covariate can be performed.

Nonparametric more than two samples. Pairwise multiple comparison tests can be requested for the "Kruskal-Wallis Test" and the "Friedman Two-Way ANOVA by Ranks." The pairwise comparisons are performed with Nemenyi's (1963) procedure. See Kirk (1968) for a description of Nemenyi's procedure.

Multiple regression (Pearson). Multiple regression can be performed with as many as 20 variables. Any variable can be designated the dependent variable and any or all of the remaining variables can be designated independent variables. WolStat can perform more than one multiple regression analysis on the same variables and place the results for all analyses in one file. A data transformation can be performed before a multiple regression analysis is performed.

\section{LEARNING AIDS}

Each of the two learning aids that are part of WolStatorthogonal comparisons and models for fixed, mixed, and random ANOVAs--has a comprehensive "Help" menu. See Wolach and McHale (1988) for a discussion of orthogonal comparisons, and see Wolach and McHale (1987) for a discussion of fixed, mixed, and random models.

\section{Orthogonal Comparisons}

Options for orthogonal comparisons. If "Orthogonal Comparisons" is selected, an investigator is provided with four options: "Show All Possible Comparisons," "Show All Possible Orthogonal Comparisons to a Comparison," "Show All Possible Orthogonal Comparisons to Comparisons Entered," and "Tutorial on Orthogonal Comparisons." The first three of the four options lead to options for three through seven treatments.

All possible comparisons. Suppose "Show All Possible Comparisons" for three treatments is selected. WolStat responds with the six possible comparisons for a three group experiment: Group 1 versus Group 2, Group 1 versus Group 3, Group 2 versus Group 3, Group 1 and Group 2 combined versus Group 3, Group 1 and Group 3 combined versus Group 2, and Group 2 and Group 3 combined versus Group 1.

All possible orthogonal comparisons to a comparison. If "Show All Possible Orthogonal Comparisons to a Comparison" for four treatments is selected, the investigator enters a comparison (e.g., Group 1 and Group 3 combined vs. Group 2 and Group 4 combined) and WolStat provides all other comparisons that are potentially orthogonal to the comparison.

All possible orthogonal comparisons to comparisons entered. If "Show All Possible Orthogonal Comparisons to Comparisons Entered" is selected, the investigator enters more than one comparison. WolStat will not accept comparisons if they are not orthogonal to each other. WolStat provides all other comparisons that are orthogonal to the comparisons that are entered.

\section{Models for Fixed, Mixed, and Random ANOVAs}

Options for fixed, mixed, and random models. If "Models for Fixed, Mixed, and Random ANOVAs" is chosen, several options appear on the screen. An investigator can choose any of the ANOVAs listed in the Appendix. Then the investigator chooses "Random" or "Fixed" for subjects and "Random" or "Fixed" separately for each of the factors. The investigator also can choose whether quasi- $F$ ratios (when necessary) should be shown with the method of Satterthwaite (1946) or that of Cochran (1951).

Output for fixed, mixed, and random models. Finally the investigator chooses which of the following should be shown on the screen: "Terms for Overall Model," "Multipliers and General Model," "Specific Model," or "F Ratios and Quasi F Ratios."

\section{CHOOSINGA STATISTICS PACKAGE}

\section{Specific Statistics}

All statistics and learning aids included in WolStat have been described or listed in the previous sections of this article. If an investigator requires a statistical implementation that can only be found in WolStat, WolStat is the obvious choice as a statistics package.

\section{Unique Advantages for a Statistics Package}

If WolStat and other statistics packages can perform a given statistic, consider residual benefits for choosing a given statistics package. For example, choose WolStat when simple main effects tests are required following an ANOVA. If WolStat and another statistics package can perform a sta- 
tistic and provide essentially the same output, consider ease of data entry. Ease of data entry includes time to enter data and familiarity with the data entry system in question.

Some investigators use more than one statistics package to perform analyses on a data set. Statistics packages with compatible data files should be chosen for data sets requiring more than one statistics package. If WolStat is one of two statistics packages used in a data analysis, the other statistics package should accept data files as text files or be able to convert proprietary data files to text files. Once a data file is converted to a text file, appropriate delimiters can be added to allow the file to be used with WolStat. The "Clean Data" features of WolStat can be used to add appropriate commas and spaces.

\section{Statistics Courses}

WolStat is particularly useful for statistics and methodology courses. It is free. Students can install it on their home computers. WolStat can be used to assist investigators (students) who are learning statistics. For example, Wolstat can be used to find significance levels for a number of statistics. It can also be used to generate Latin squares, provide random numbers with varying constraints, demonstrate orthogonal comparisons, and demonstrate differences in fixed, mixed, and random models.

\section{AVAILABILITY, INSTALLATION, AND UPDATES}

\section{How to Obtain WolStat}

Request a copy of WolStat from wolach@iit.edu. Include an e-mail address and a mailing address. Directions for finding the Web page for obtaining WolStat will be returned by e-mail. If a potential user wants a CD with WolStat and an automatic installation program, the $\mathrm{CD}$ can be sent by regular mail. Directions for installing WolStat and the manual for WolStat are in the "Readme" file on the CD and on the Web page. The license for WolStat is free and open source.

\section{Size of WolStat}

When WolStat is installed on a computer, it takes under $5 \mathrm{MB}$ of hard disk space. The file for the manual is in the main folder of the CD, the WolStat directory on the hard drive, and on the Web page. Virtually any text editor or word processor can be used to print the manual. The manual can also be installed with an icon on the "Start" menu. The icon for the manual can be clicked and the manual can be read in a text box that appears on the monitor. The "Help" system of WolStat contains virtually all of the information found in the manual.

\section{Source Code}

The main folder of the WolStat CD has seven foldersWolStat1, WolEdit, Correl, tANOCOV, OrModHp, FiveWay, and HelpIt-that contain the Visual Basic 5 files for the seven programs used by WolStat. These folders are also on the Web page. If a user does not intend to modify the statistics package, the files in the seven folders can be ignored.

If a user wants to modify one of the seven Wolstat programs, the user should place the files in one of the seven folders on the $C D$ in a folder on the hard drive of a computer. Visual Basic 5 (or 6) can be used to load the project (.vbp) file for the program.

\section{Modifying WolStat}

Feel free to modify the program files, recompile the files, and distribute your version of the program to potentially interested users. We would appreciate receiving copies of the modified program.

Since the program is a Windows program, it may take some time for program users to integrate their own additions into the program. We would be happy to make additions to the program work from the menu system of WolStat. Send potential additions to the first author by e-mail or regular mail. Include Visual Basic code, references to the statistic in question (journal article, book, etc.). Provide a suggestion for where the addition should be placed in the menu system. The code need not include any attempt at code required to make the program work from the WolStat menu system.

We would also be willing to attempt to code some procedures for interested users. Supply a complete copy of the section of a book (or complete journal article) showing formulas and an example of calculations for the procedure.

All program users who send code should indicate whether the code can be included in our version of WolStat.

\section{AUTHOR NOTE}

Correspondence concerning this article should be addressed to A. $H$. Wolach, Institute of Psychology, Illinois Institute of Technology, Chicago, IL 60616 (e-mail: wolach@iit.edu).

\section{REFERENCES}

Cochran, W. G. (1951). Testing a linear relation among variances. Biometrics, 7, 17-32.

KIRK, R. E. (1968). Experimental design: Procedures for the behavioral sciences. Belmont, CA: Brooks/Cole.

KIRK, R. E. (1995). Experimental design: Procedures for the behavioral sciences (3rd ed.). Pacific Grove, CA: Brooks/Cole.

NeMENYI, P. (1963). Distribution-free multiple comparisons. Unpublished doctoral dissertation, Princeton University, Princeton, NJ.

SATTERTHWAITE, F. E. (1946). An approximate distribution of estimates of variance components. Biometrics Bulletin, 2, 110-114.

WinER, B. J., BROWN, D. R., \& Michels, K. M. (1991). Statistical principles in experimental design (3rd ed.). New York: McGraw-Hill.

WOLACH, A. H., \& MCHALE, M. A. (1987). $F$ ratios and quasi $F$ ratios for fixed, mixed, and random model ANOVAs. Behavior Research Methods, Instruments, \& Computers, 19, 409-412.

Wolach, A. H., \& McHALE, M. A. (1988). Orthogonal comparisons. Behavior Research Methods, Instruments, \& Computers, $20,337$. 
APPENDIX

Statistics Requiring a Data File

\begin{tabular}{|c|c|c|}
\hline Category & Subcategory & $\begin{array}{c}\text { Data Transformations } \\
\text { Possible } \\
\end{array}$ \\
\hline$t$ Test & $\begin{array}{l}\text { One-sample } \\
\text { Independent groups } \\
\text { Correlated measures }\end{array}$ & $\begin{array}{l}\text { Yes } \\
\text { Yes } \\
\text { Yes }\end{array}$ \\
\hline Independent Groups ANOVA & $\begin{array}{l}\text { One-way (CR-p) } \\
\text { Two-way (CRF-pq) } \\
\text { Three-way (CRF-pqr) } \\
\text { Four-way (CRF-pqrs) } \\
\text { Five-way (CRF-pqrst) }\end{array}$ & $\begin{array}{l}\text { Yes } \\
\text { Yes } \\
\text { Yes } \\
\text { Yes } \\
\text { Yes }\end{array}$ \\
\hline Split Plot Factorial & $\begin{array}{l}\text { Two-way (SPF-p.q) } \\
\text { Three-way (SPF-pq.r) } \\
\text { Three-way (SPF-p.qr) } \\
\text { Four-way (SPF-pqr.s) } \\
\text { Four-way (SPF-pq.rs) } \\
\text { Four-way (SPF-p.qrs) } \\
\text { Five-way (SPF-pqrs.t) } \\
\text { Five-way (SPF-pqr.st) } \\
\text { Five-way (SPF-pq.rst) } \\
\text { Five-way (SPF-p.qrst) }\end{array}$ & $\begin{array}{l}\text { Yes } \\
\text { Yes } \\
\text { Yes } \\
\text { Yes } \\
\text { Yes } \\
\text { Yes } \\
\text { Yes } \\
\text { Yes } \\
\text { Yes } \\
\text { Yes }\end{array}$ \\
\hline $\begin{array}{l}\text { Randomized Block and Random- } \\
\text { ized Block Factorial ANOVA }\end{array}$ & $\begin{array}{l}\text { One-way (RB-.p) } \\
\text { Two-way (RBF-.pq) } \\
\text { Three-way (RBF-.pqr) } \\
\text { Four-way (RBF-.pqrs) } \\
\text { Five-way (RBF-.pqrst) }\end{array}$ & $\begin{array}{l}\text { Yes } \\
\text { Yes } \\
\text { Yes } \\
\text { Yes } \\
\text { Yes }\end{array}$ \\
\hline Latin Square ANOVA LSK-p & ([no subcategory]) & Yes \\
\hline Independent Groups ANOCOVA & $\begin{array}{l}\text { One-way (CRAC-p, } 1 \text { cov.) } \\
\text { One-way (CRAC-p, } 2 \text { cov.) } \\
\text { Two-way (CRFAC-pq, } 1 \text { cov.) } \\
\text { Three-way (CRFAC-pqr, } 1 \text { cov.) }\end{array}$ & $\begin{array}{l}\text { Yes } \\
\text { Yes } \\
\text { Yes } \\
\text { Yes }\end{array}$ \\
\hline Split Plot Factorial ANOCOVA & $\begin{array}{l}\text { Two-way (SPFAC-p.q, } 1 \text { cov.) } \\
\text { Three-way (SPFAC-pq.r, } 1 \text { cov.) } \\
\text { Three-way (SPFAC-p.qr, } 1 \text { cov.) }\end{array}$ & $\begin{array}{l}\text { Yes } \\
\text { Yes } \\
\text { Yes }\end{array}$ \\
\hline $\begin{array}{l}\text { Randomized Block and Random- } \\
\text { ized Block Factorial ANOCOVA }\end{array}$ & $\begin{array}{l}\text { One-way (RBAC-.p, } 1 \text { cov.) } \\
\text { Two-way (RBFAC-.pq, } 1 \text { cov.) } \\
\text { Three-way (RBFAC-.pqr } 1 \text { cov.) }\end{array}$ & $\begin{array}{l}\text { Yes } \\
\text { Yes } \\
\text { Yes }\end{array}$ \\
\hline Latin Square ANOCOVA LSAC-p & ([no subcategory]) & Yes \\
\hline $\begin{array}{l}\text { Nonparametric } 2 \times 2 \text { and One } \\
\text { Sample }\end{array}$ & $\begin{array}{l}\text { Fisher exact probability test } \\
\text { McNemar test } \\
\text { Multiple category and one-sample chi-square } \\
\text { One-sample runs test } \\
\text { Kolmogorov-Smirnov one-sample test } \\
\text { One-sample randomization test } \\
\text { Change-point test (continuous variable) } \\
\text { Change-point test (binomial variable) } \\
\text { Test for distributional symmetry }\end{array}$ & $\begin{array}{l}\text { No } \\
\text { No } \\
\text { No } \\
\text { No } \\
\text { No } \\
\text { No } \\
\text { No } \\
\text { No } \\
\text { No }\end{array}$ \\
\hline Nonparametric Two Samples & $\begin{array}{l}\text { Mann-Whitney } U \text { test } \\
\text { Two-sample median test } \\
\text { Sign test } \\
\text { Wilcoxon sign ranks test } \\
\text { Kolmogorov-Smirnov two-sample test } \\
\text { Wald-Wolfowitz runs test } \\
\text { Moses test of extreme reactions } \\
\text { Two-sample randomization test } \\
\text { Randomization test (correlated) } \\
\text { Walsh test } \\
\text { Siegel-Tukey test } \\
\text { Permutation test, independent groups }\end{array}$ & $\begin{array}{l}\text { No } \\
\text { No } \\
\text { No } \\
\text { No } \\
\text { No } \\
\text { No } \\
\text { No } \\
\text { No } \\
\text { No } \\
\text { No } \\
\text { No } \\
\text { No }\end{array}$ \\
\hline
\end{tabular}


APPENDIX (Continued)

\begin{tabular}{lll}
\hline & Permutation test, correlated & No \\
Nonparametric More Than Two & Robust rank order test & No \\
Samples & Kruskal-Wallis test & No \\
& Wilcoxon one-way ANOVA by ranks & No \\
& Multiple sample median test & No \\
& Friedman two-way ANOVA by ranks & No \\
& Cochran Q test & No \\
& Chi-square (two-way) & No \\
& Page test for ordered alternatives & No \\
& Jonckhere test for ordered alternatives & No \\
& Quade ANOCOVA & No \\
& Pearson product moment correlation & Yes \\
Correlation & Pearson partial and second order & Yes \\
& Kendall's tau & No \\
& Tau partial and second order & No \\
& Spearman rank order correlation coefficient & No \\
& Kendall coefficient of concordance $(W)$ & No \\
& Biserial correlation coefficient & No \\
& Point biserial correlation coefficient & No \\
& Cohen's kappa & No \\
& Contingency coefficient $(C)$ & No \\
& Phi coefficient & No \\
& Eta correlation ratio & No \\
& Cramer coefficient for degree of association & No \\
& Gamma statistic & No \\
& Lambda for asymmetrical association & No \\
& Somers' statistic $(d)$ & No \\
& ([no subcategory]) & Yes \\
\hline
\end{tabular}

(Manuscript received January 24, 2007;

revision accepted for publication June 4,2007 .) 